\title{
MAPPIT: a versatile tool to study cytokine receptor signalling
}

\author{
Irma Lemmens" $\dagger$, Sam Lievens" $\dagger$ and Jan Tavernier" ${ }^{1}$ \\ "Department of Medical Protein Research, VIB, Ghent University, A. Baertsoenkaai 3, 9000 Ghent, Belgium, and †Department of Biochemistry, \\ Faculty of Medicine and Health Sciences, Ghent University, A. Baertsoenkaai 3, 9000 Ghent, Belgium
}

\begin{abstract}
MAPPIT (mammalian protein-protein interaction trap) is a cytokine receptor-based two-hybrid method that operates in intact mammalian cells. A bait is fused C-terminally to a STAT (signal transducer and activator of transcription) recruitment-deficient receptor, whereas the prey is linked to functional STATbinding sites. When bait and prey interact a ligand-dependent complementation of the STAT recruitment deficiency occurs, leading to activation of a STAT-responsive reporter. MAPPIT is very well suited to study protein interactions involving activated cytokine receptors as the technique allows modification of the bait protein in a physiologically optimal environment.
\end{abstract}

\section{Cytokines and their receptors}

Cytokines are small messenger molecules that are involved in cell-cell communication and are critical to many vital processes such as embryonic development, growth, haemopoiesis and immune responses. They cannot cross the cellular membrane but make use of transmembrane receptors that capture cytokines and transmit their signal inside the cell. In this mini-review, we will mainly focus on homomeric type I cytokine receptors such as the EpoR (erythropoietin receptor), GHR (growth hormone receptor), LR (leptin receptor) and GCSFR (granulocyte colony-stimulating factor receptor). These cytokine receptors have no intrinsic kinase activity but make use of associated JAKs (Janus kinases). Upon ligand binding the JAKs are brought into close proximity enabling them to cross-phosphorylate each other. Subsequently the JAKs phosphorylate tyrosine residues in the cytoplasmic receptor tail, thereby creating docking sites for signalling molecules such as STAT (signal transducer and activator of transcription) proteins. After binding of these STATs they are phosphorylated in turn by the JAKs and translocate as dimers to the nucleus where they initiate transcription of target genes (Figure 1A). Several mechanisms exist to modulate and terminate cytokine signalling. Among these, the SOCS (suppressor of cytokine signalling) protein

Key words: cytokine receptor, mammalian protein-protein interaction trap (MAPPIT), signal transducer and activator of transcription (STAT), signal transduction, suppressor of cytokine signalling (SOCS), Toll-like receptor (TLR).

Abbreviations used: $\beta c$, beta common; Epo, erythropoietin; EpoR, Epo receptor; GCSFR, granulocyte colony-stimulating factor receptor; GHR, growth hormone receptor; GM-CSF, granulocyte/macrophage colony-stimulating factor; gp 130, glycoprotein 130; HEK-293T cells, human embryonic kidney cells expressing the large T-antigen of SV40 (simian virus 40), IRAK, interleukin-1-receptor-associated kinase; IRS4, insulin receptor substrate 4; JAK, Janus kinase; LR, leptin receptor; MAPPIT, mammalian protein-protein interaction trap; MyD88, myeloid differentiation primary response protein 88; Mal, MyD88 adaptor-like protein; PLC $\gamma$ phospholipase $\mathrm{C} \gamma$; SH2, Src homology 2; CIS, cytokine-inducible SH2 domain-containing protein SOCS, suppressor of cytokine signalling; STAT, signal transducer and activator of transcription; TIR, Toll/interleukin-1 receptor; TRIF, TIR-domain-containing adaptor inducing interferon- $\beta$; TLR, Toll-like receptor.

${ }^{1}$ To whom correspondence should be addressed (email jan.tavernier@ugent.be). family plays a prominent role. These proteins are rapidly induced upon cytokine receptor activation and are recruited to the activated receptor complexes. They exhibit multiple inhibitory modes including blocking of STAT binding, inhibition of JAK activity or proteasomal targeting of the receptor complex (for reviews, refer to [1-4]).

\section{MAPPIT (mammalian protein-protein interaction trap)}

MAPPIT is a two-hybrid method that operates in intact mammalian cells and is based on the JAK/STAT pathway described above. The original MAPPIT approach as shown in Figure 1(B) makes use of a chimaeric receptor consisting of the extracellular domain of the human EpoR, which is a wellcharacterized homodimeric receptor, and the transmembrane and cytoplasmic tail of the long-form LR. The LR contains only three conserved tyrosine residues in its cytoplasmic tail, at amino acid positions 985, 1077 and 1138. To prevent the recruitment of STAT3 and SOCS molecules, all three tyrosine residues were mutated to phenylalanine. A bait is then fused C-terminally to this STAT recruitment-deficient chimaeric cytokine receptor. The prey is linked to functional STAT recruitment sites from the gp130 (glycoprotein 130) receptor chain. When bait and prey interact, ligand-dependent complementation of the STAT recruitment deficiency occurs, leading to activation of a STAT-responsive reporter [5].

Instead of making use of a chimaeric cytokine receptor, the full-length LR can be used as well, with only Tyr-1138 being mutated to phenylalanine to prevent STAT3 recruitment (Figure 1C). This way, protein interactions can be studied with the LR in its normal oligomeric configuration [6]. In another MAPPIT setup, we can use the wild-type EpoR (Figure 1D) [7]. Since the EpoR signals via STAT5 and not STAT3 and since the levels of STAT5 are low in the commonly used HEK293T cells [human embryonic kidney cells expressing the 
Figure 1 | JAK/STAT signalling and alternative MAPPIT setups

(A) Layout of the JAK/STAT signalling pathway. Binding of a cytokine ligand (not shown) to a CR (cytokine receptor) leads to activation of the associated JAK tyrosine kinases. These phosphorylate tyrosine residues ( $Y$ ) in the cytosolic tail of the receptor thus providing docking sites for STAT proteins. STATs in turn are activated by the JAKs and relocalize as dimers to the nucleus leading to stimulation of transcription of their target genes. (B) In the original MAPPIT set-up, a bait protein (B) is C-terminally fused to a STAT recruitment-deficient receptor consisting of the extracellular domain of the EpoR and the transmembrane and intracellular portion of an LR wherein all tyrosine residues are replaced by phenylalanine (F) residues. When co-expressed with an interacting prey (P) that is fused to a gp130 receptor fragment containing functional STAT3 recruitment sites, signalling is restored and measured using a STAT3-responsive reporter. (C) Using the full-length LR itself as bait allows dissection of the interaction pattern of intracellular tyrosine residues in the receptor tail. The most distal Tyr-1138 is mutated to prevent direct signalling of the LR 'bait'. (D) As for the LR, also the full-length EPOR can be used as a bait to analyse proteins interacting with the receptor. (E) As only the membrane proximal region of the LR intracellular tail which is involved in binding JAK2 is essential, the rest of the cytosolic domain can be replaced by a flexible linker containing repeated GGS (Gly-Gly-Ser) units. This allows the study of specific tyrosine motifs of the LR intracellular region. (F) In haemopoietic cells, which express no or only very low levels of STAT3, STAT5 is used as a mediator of the MAPPIT signal. To this end, preys are coupled to a fragment of the $\beta$ c receptor containing functional STAT5 recruitment sites instead of a fragment of the gp130 receptor. (G) In the heteromeric MAPPIT assay, the extracellular domains of the GM-CSF receptor are used to obtain a heteromeric receptor cluster. As in the basic MAPPIT setup, the tyrosine $\rightarrow$ phenylalanine-mutated transmembrane and intracellular domains of the LR are used. A modifying enzyme is attached to one of the resulting receptor chains [GMR $\alpha$ (GM-CSF receptor $\alpha)$-LR], and the bait is fused to the other ( $\beta C$-LR). This method allows the use of enzymatically modified baits.
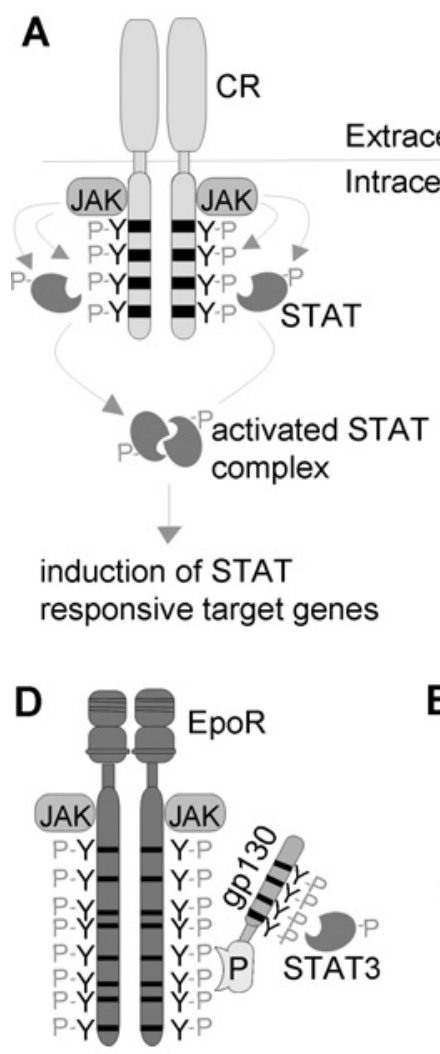

B

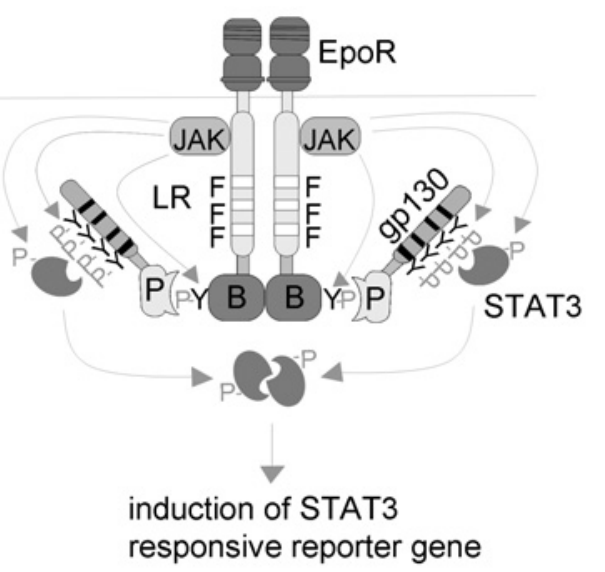

C

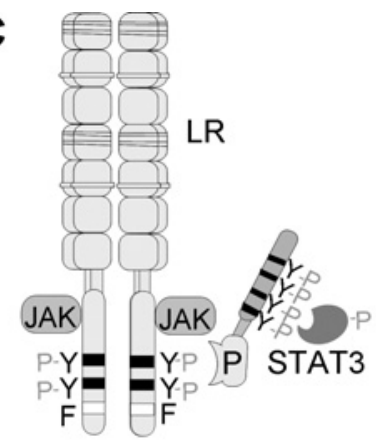

E

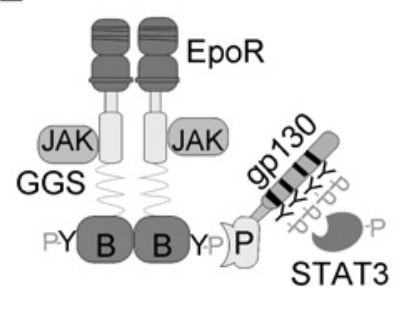

$\mathbf{F}$

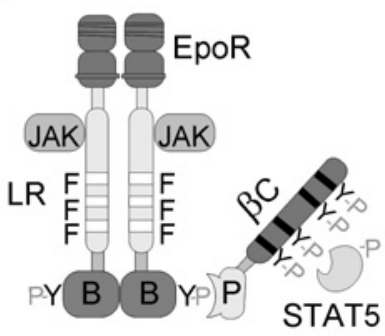

G $\beta C$

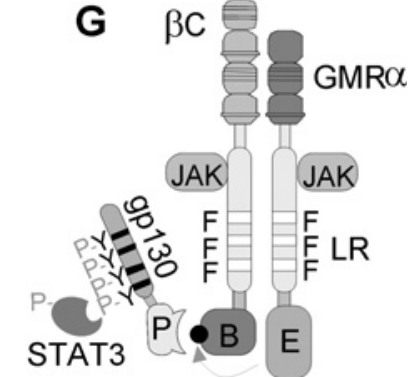

large T-antigen of SV40 (simian virus 40)], no reporter activation can be detected after stimulation with Epo (erythropoietin) allowing the use of the EpoR itself and mutants thereof as bait. To study the isolated tyrosine motifs of the LR, we replaced the LR cytosolic domain following the membraneproximal box1 motif (which is needed for the association of the JAK kinases) by flexible Gly-Gly-Ser repeats (Figure 1E) [6]. To be able to use MAPPIT in haemopoietic cells that have no or very low STAT3 levels, $\beta \mathrm{c}$ (beta common) MAPPIT was created, which employs STAT5 as a signal transducer. To accomplice this, the prey was fused to a portion of the $\beta \mathrm{c}$ chain containing STAT5 recruitment sites (Figure 1F) [8].

\section{Analytical use of MAPPIT}

An intrinsic feature of the MAPPIT technology is that the bait can be tyrosine-phosphorylated by the receptor-associated 
JAKs. This makes MAPPIT extremely useful for the study of protein interactions involving cytokine receptors, since many interactions depend on modification by the JAKs. The different MAPPIT set-ups (Figures $1 \mathrm{~B}-1 \mathrm{~F}$ ) were used to identify and study interactions of signalling molecules involved in various signalling cascades initiated by homomeric type I cytokine receptors. A brief overview is given below.

\section{EpoR}

Epo is essential for red blood cell development. Binding of Epo to its receptor prevents apoptosis of erythroid progenitors and promotes proliferation and erythroid maturation. To be able to determine the binding site of different signalling molecules with the EpoR, we used intracellular segments of the EpoR containing one or two tyrosine residues as bait. Several signalling molecules served as prey including CIS [cytokine-inducible SH2 (Src homology 2)-domaincontaining protein], SOCS2, PLC $\gamma$ (phospholipase $\mathrm{C} \gamma$ ), STAT5 $a$ and STAT5b. Interestingly, the STAT5a and STAT5b interaction sites were identical and overlapped with the binding sites of CIS and SOCS2, indicating that CIS and SOCS2 can inhibit STAT5 activation by blocking its recruitment site. CIS and SOCS2 bound additional, although not completely similar, tyrosine motifs, illustrating a differential binding between these two highly related SOCS proteins. The binding sites of PLC $\gamma$ were also identified and mutational analysis revealed the relevant PLC $\gamma$ interaction domain. The binding sites of CIS, SOC2 and PLC $\gamma$ were confirmed using the wildtype EpoR as bait. By mutating tyrosine residues in the wild-type EpoR the essential docking sites were identified. Most of the MAPPIT analyses were performed in HEK-293T cells but subsets were confirmed in premyeloid TF-1 cells, a more natural setting of the EpoR. Furthermore, EMSA (electrophoretic mobility-shift assay) experiments showed that a wild-type STAT5 prey after binding to its EpoR bait motif could still translocate to the nucleus to bind DNA, implying that MAPPIT can detect transient interactions [7].

\section{GHR}

GH (growth hormone) is a pleiotropic hormone with an important role in the regulation of postnatal growth. It regulates carbohydrate, lipid, nitrogen and mineral metabolism and can stimulate differentiation and mitogenesis of a variety of cell types in different tissues. As for the EpoR, the tyrosine motifs in its cytoplasmic tail were separately cloned as bait. In striking contrast to the EpoR, the binding sites for CIS and SOCS2 completely segregated from the STAT5 $a$ and -b recruitment sites, ruling out SOCS-mediated inhibition of STAT5 activation by competing for shared binding sites [9].

\section{LR}

Leptin is critical for body weight homoeostasis by balancing food intake and energy expenditure. It is also involved in growth, reproduction, immune regulation, bone formation and angiogenesis. MAPPIT analysis, both in HEK-293T and TF-1 cells, using the mutated LR and its separate tyrosine motifs as bait (Figures $1 \mathrm{C}$ and $1 \mathrm{E}$ ), revealed that CIS interacts with Tyr-985 and Tyr-1077, whereas SOCS2 and STAT5a only interact with Tyr-1077. Wild-type SOCS2 easily interferes with binding of the CIS and STAT5a prey at the Tyr1077 motif, indicating that SOCS2 binds with higher affinity. Remarkably, although SOCS2 does not bind the Tyr-985 motif, it can interfere with the binding of CIS to this site. This unexpected finding could be explained by the direct binding of SOCS2 on the CIS SOCS box, thereby targeting it for proteasomal degradation $[6,10]$. More detailed analysis using CIS mutants revealed that CIS binding to the LR and EpoR not only depends on its SH2 domain but also on its SOCS box, with a critical role for the CIS Tyr-253 and the Elongin $\mathrm{B} / \mathrm{C}$ recruitment site. Much in contrast, the SOCS box of SOCS2 does not play a role in substrate binding [11,12].

Using the $\beta$ c setup (Figure $1 \mathrm{~F}$ ), we confirmed the binding of CIS, SOCS2 and STAT5a to the Tyr-1077 motif in the murine $\mathrm{Ba} / \mathrm{F} 3$ pro- $\mathrm{B}$ cell line and revealed that, in addition, PLC $\gamma$, SOCS6 and SOCS7 also interact with the Tyr-1077 motif [8]. More recently, MAPPIT experiments in both HEK-293T and in the physiologically highly relevant N38 hypothalamic cell line revealed that IRS4 (insulin receptor substrate 4) interacts with the Tyr-1077 motif. Using IRS4 as bait, we confirmed the interaction with the p85 subunit of PI3K (phosphoinositide 3-kinase), SOCS6 and SOCS7 and documented interactions with PLC $\gamma$ and SOCS2 [13].

\section{GCSFR}

The GCSFR is involved in neutrophil development by activating pathways that control the proliferation and survival of myeloid precursors. Using MAPPIT, we confirmed the tyrosine-phosphorylation dependent interaction of SHC (SH2-domain-containing transforming protein), Grb2 (growth factor receptor-bound protein 2) and Wsb-2 (WD40-repeat and SOCS box containing protein 2) with the cytoplasmic tail of the GCSFR [14].

\section{TLR (Toll-like receptor)}

The application of MAPPIT as a tool to dissect protein interactions is not limited to cytokine receptor signalling. We recently reported the detailed MAPPIT analysis of the signalling events downstream of TLR activation $[15,16]$. TLRs are activated when cells encounter pathogen-derived structures such as lipopolysaccharide, flagellin or dsRNA and the signalling pathways they trigger lead to a first line of defence as part of the innate immune response. Activated TLRs couple to downstream signalling cascades through a set of five adaptor molecules MyD88 (myeloid differentiation primary response protein 88 ), Mal (MyD88 adaptor-like protein), TRIF [TIR (Toll/interleukin-1 receptor)-domaincontaining adaptor inducing interferon- $\beta$ ], TRAM (TRIFrelated adaptor molecule) and SARM [sterile $\alpha$ and HEAT (huntingtin, elongation factor 3 , the PR65/A subunit of 
protein phosphatase $2 \mathrm{~A}$ and the lipid kinase Tor)-Armadillo motifs containing protein]\} which in part determine the specificity of the response [17]. Combinations of TLR intracellular domains and TLR adaptors cloned as bait and/or prey were tested in MAPPIT, identifying novel interactions and confirming previously documented interactions such as the homo- and hetero-dimerization of MAL and MyD88 [15]. Interestingly, whereas the TIR domain was sufficient for heterodimerization, the formation of homodimers of both MyD88 and MAL also depended on other subdomains. Intriguingly, although the role of MyD88 in TLR4 signalling is well established, no interaction could be detected between TLR4 and this adaptor. Co-expression of MAL however enabled a clear MAPPIT signal, indicating that binding between MyD88 and TLR4 is indirect and that MAL bridges this interaction. MAPPIT was further applied in pathway walking along one of the TLR4 signalling cascades starting with TLR4 dimerization, and through recruitment of MAL and MyD88 leading to association of IRAK (interleukin-1receptor-associated kinase)-4 and IRAK-1 [16]. The MyD88independent pathway starting from TLR4 and mediated by TRIF could not be analysed using MAPPIT as no signal was obtained with TRIF as bait or prey. Immunofluorescence analysis of TRIF prey revealed that the fusion protein was localized to a perinuclear compartment, probably restricting it from interacting with the membrane-proximal bait proteins.

\section{Heteromeric MAPPIT}

As shown above, MAPPIT can successfully detect modification-independent and tyrosine phosphorylation-dependent interactions. Protein modification in this approach however strictly depends on the receptor-associated JAKs. To allow the detection of other modification-dependent interactions, heteromeric MAPPIT was developed. In this variant, the homodimeric extracellular domain of the EpoR is replaced by the extracellular parts of the heteromeric GM-CSF (granulocyte/macrophage colony-stimulating factor) receptor allowing fusion of a modifying enzyme to one receptor chain and the bait to the other chain (Figure 1G). Using this method, we were able to detect serine phosphorylation-dependent interactions in TGF- $\beta$ R (transforming growth factor- $\beta$ receptor) family signalling [18].

\section{Conclusion}

Using different MAPPIT set-ups (Figures 1B-1G) in different cell lines the recruitment of various signalling and adaptor molecules to activated receptors was studied in greater detail. New roles for SOCS proteins and for their prototypical SOCS box were elucidated. This shows that the near-optimal physiological context makes MAPPIT very well suited for the analysis of receptor signalling. Its use is however not limited to analysing signalling cascades. We have observed MAPPIT signals for protein-protein interactions involved in a wide range of cellular functions including transcriptional regulation, cell cycle control, apoptosis, antiviral defence and so on. This warrants the use of MAPPIT in large-scale interactome mapping programs [19].

\section{References}

1 Alexander, W.S. and Hilton, D.J. (2004) The role of suppressors of cytokine signaling (SOCS) proteins in regulation of the immune response. Annu. Rev. Immunol. 22, 503-529

2 Johnston, J.A. (2004) Are SOCS suppressors, regulators, and degraders? J. Leukocyte Biol. 75, 743-748

3 O'Shea, J.J. and Murray, P.J. (2008) Cytokine signaling modules in inflammatory responses. Immunity 28, 477-487

4 Yoshimura, A., Naka, T. and Kubo, M. (2007) Socs proteins, cytokine signalling and immune regulation. Nat. Rev. Immunol. 7, 454-465

5 Eyckerman, S., Verhee, A., van der Heyden, J., Lemmens, I., Ostade, X., Vandekerckhove, J. and Tavernier, J. (2001) Design and application of a cytokine-receptor-based interaction trap. Nat. Cell Biol. 3, 1114-1119

6 Lavens, D., Montoye, T., Piessevaux, J., Zabeau, L., Vandekerckhove, J., Gevaert, K., Becker, W., Eyckerman, S. and Tavernier, J. (2006) A complex interaction pattern of CIS and SOCS2 with the leptin receptor. J. Cell Sci. 119, 2214-2224

7 Montoye, T., Lemmens, I., Catteeuw, D., Eyckerman, S. and Tavernier, J. (2005) A systematic scan of interactions with tyrosine motifs in the erythropoietin receptor using a mammalian 2-hybrid approach. Blood 105, 4264-4271

8 Montoye, T., Piessevaux, J., Lavens, D., Wauman, J., Catteeuw, D., Vandekerckhove, J., Lemmens, I. and Tavernier, J. (2006) Analysis of leptin signalling in hematopoietic cells using an adapted MAPPIT strategy. FEBS Lett. 580, 3301-3307

9 Uyttendaele, I., Lemmens, I., Verhee, A., De Smet, A.S., Vandekerckhove, J., Lavens, D., Peelman, F. and Tavernier, J. (2007) Mammalian protein-protein interaction trap (MAPPIT) analysis of STAT5, CIS, and SOCS2 interactions with the growth hormone receptor. Mol. Endocrinol. 21, 2821-2831

10 Piessevaux, J., Lavens, D., Montoye, T., Wauman, J., Catteeuw, D., Vandekerckhove, J., Belsham, D., Peelman, F. and Tavernier, J. (2006) Functional cross-modulation between SOcS proteins can stimulate cytokine signaling. J. Biol. Chem. 281, 32953-32966

11 Lavens, D., Ulrichts, P., Catteeuw, D., Gevaert, K., Vandekerckhove, J., Peelman, F., Eyckerman, S. and Tavernier, J. (2007) The C-terminus of CIS defines its interaction pattern. Biochem. J. 401, 257-267

12 Piessevaux, J., De Ceuninck, L., Catteeuw, D., Peelman, F. and Tavernier, J. (2008) Elongin B/C recruitment regulates substrate binding by CIS. J. Biol. Chem. 283, 21334-21346

13 Wauman, J., De Smet, A.S., Catteeuw, D., Belsham, D. and Tavernier, J. (2008) Insulin receptor substrate 4 couples the leptin receptor to multiple signaling pathways. Mol. Endocrinol. 22, 965-977

14 Erkeland, S.J., Aarts, L.H., Irandoust, M., Roovers, O., Klomp, A., Valkhof, M., Gits, J., Eyckerman, S., Tavernier, J. and Touw, I.P. (2007) Novel role of WD40 and SOCS box protein-2 in steady-state distribution of granulocyte colony-stimulating factor receptor and $\mathrm{G}$-CSF-controlled proliferation and differentiation signaling. Oncogene 26, 1985-1994

15 Ulrichts, P., Peelman, F., Beyaert, R. and Tavernier, J. (2007) MAPPIT analysis of TLR adaptor complexes. FEBS Lett. 581, 629-636

16 Ulrichts, P. and Tavernier, J. (2008) MAPPIT analysis of early Toll-like receptor signalling events. Immunol. Lett. 116, 141-148

17 O'Neill, L.A. and Bowie, A.G. (2007) The family of five: TIR-domaincontaining adaptors in Toll-like receptor signalling. Nat. Rev. Immunol. 7, 353-364

18 Lemmens, I., Eyckerman, S., Zabeau, L., Catteeuw, D., Vertenten, E., Verschueren, K., Huylebroeck, D., Vandekerckhove, J. and Tavernier, J. (2003) Heteromeric MAPPIT: a novel strategy to study modificationdependent protein-protein interactions in mammalian cells. Nucleic Acids Res. 31, e75

19 Yu, H., Braun, P., Yildirim, M.A., Lemmens, I., Venkatesan, K., Sahalie, J., Hirozane-Kishikawa, T., Gebreab, F., Li, N., Simonis, N. et al. (2008) High-quality binary protein interaction map of the yeast interactome network. Science 322, 104-110

Received 8 August 2008

doi:10.1042/BST0361448 